\title{
Cyclocarya paliurus (Batal.) Iljinskaja polysaccharides alleviate type 2 diabetes mellitus in rats by resisting inflammatory response and oxidative stress
}

\author{
Wensheng YAN ${ }^{1 *}$ (D), Lingjun JIANG ${ }^{1}$, Jifen $\mathrm{XU}^{1}$
}

\begin{abstract}
This study aimed to investigate the effects of Cyclocarya paliurus (Batal.) Iljinskaja polysaccharides (CPP) on the glucose-lipid metabolism in T2DM rats. T2DM model was established in 40 Wistar rats which were then randomly divided into model, CPP-1, CPP-2 and CPP-3 groups. Ten normal rats were selected as the control group. The CPP-1, CPP-2 and CPP-3 group were intragastrically administrated with 100, 200 and $400 \mathrm{mg} / \mathrm{kg}$ CPP for 4 weeks, respectively. Results showed that, during the treatment, compared with model group, in CPP treatment groups the T2DM symptoms were lightened, and the body weight was improved. After treatment, compared with model group, in CPP-3 group the blood fasting plasma glucose, fasting insulin and glycosylated hemoglobin levels were reduced $(\mathrm{P}<0.05)$, the blood total cholesterol, triglyceride and low-density lipoprotein cholesterol levels were decreased $(\mathrm{P}<0.05)$, the serum $\mathrm{C}$-reactive protein, tumor necrosis factor $a$ and interleukin 6 levels were decreased $(\mathrm{P}<0.05)$, the serum malondialdehyde level was reduced $(\mathrm{P}<0.05)$, and the serum superoxide dismutase and glutathione peroxidase levels were increased $(\mathrm{P}<0.05)$. In conclusion, $\mathrm{CPP}$ can alleviate the T2DM in rats, which may be related to its resisting inflammatory response and oxidative stress in body.
\end{abstract}

Keywords: Cyclocarya paliurus (Batal.) Iljinskaja; polysaccharides; type 2 diabetes mellitus; inflammatory response; oxidative stress.

Practical Application: Cyclocarya paliurus (Batal.) Iljinskaja polysaccharides may have application value for clinical treatment of type 2 diabetes mellitus.

\section{Introduction}

Type 2 diabetes mellitus (T2DM) is a non-insulin dependent diabetes mellitus, which is caused by the decrease in glucose metabolism regulation ability of insulin accompanied by islet $\beta$ cell dysfunction (Olokoba et al., 2012). Insulin resistance and insulin secretion disorder are the main pathological features of T2DM, but the specific mechanism is still unclear (Cavaghan et al., 2000). In recent years, studies have shown that T2DM is a low-grade chronic inflammatory disease. The cytokine-mediated inflammatory response plays an important role in the impaired glucose tolerance and T2DM, and the concentration of a variety of inflammatory factors will increase with the progress of the disease (Diakakis et al., 2005; van Doorn et al., 2006; Ruotsalainen et al., 2006; Tatsch et al., 2015). In addition, the oxidative stress is closely related to the insulin resistance and insulin secretion dysfunction which determine the progress of T2DM (Tangvarasittichai, 2015; Rehman \& Akash, 2017). Cyclocarya paliurus (Batal.) Iljinskaja belongs to the dicotyledneae family. It is one of the unique species of plants in China, and is also one of the most endangered plants. It is found that, Cyclocarya paliurus (Batal.) Iljinskaja can be used to effectively control diabetes (Kurihara et al., 2003), hypertension (Huang et al., 1986) and hyperlipidemia (Nan et al., 2015). Polysaccharides are the main physiological active substance in Cyclocarya paliurus (Batal.) Iljinskaja, which have aroused great interests and widespread concerns (Xie et al., 2010; Xie et al., 2014;).
In recent years, more and more attention has been paid to the study of functional factors and the development of functional food. Therefore, developing Cyclocarya paliurus (Batal.) Iljinskaja polysaccharides (CPP) and investigating their activities are of great practical significance. This study investigated the effect of CPP on the glucose-lipid metabolism in T2DM rats and the mechanisms related to inflammatory response and oxidative stress. The objective was to provide an experimental basis for further studying the clinical therapeutic effects of CPP on T2DM.

\section{Materials and methods}

\subsection{Materials}

The whole plant of Cyclocarya paliurus (Batal.) Iljinskaja was purchased from Yichang Qingqianliu Biological Technology Co., Ltd. (Yichang, China). Male Wistar rats weighing 180-200 g were obtained from the Guangdong Medical Laboratory Animal Center (Guangzhou, China). AB-8 macroporous adsorption resin was provided by Chemical Plant of Nankai University (Tianjin, China). Streptozotocin was provided by Shanghai Maokang Biotechnology Co., Ltd. (Shanghai, China). The kits relevant for detection of blood glucose, blood lipid and inflammatory factor and other reagents were purchased from Sigma-Aldrich Corp. (MO, USA). 


\section{Preparation of CPP}

Cyclocarya paliurus (Batal.) Iljinskaja was washed and dried $60{ }^{\circ} \mathrm{C}$ for $10 \mathrm{~h}$, followed by pulverizing. The $500 \mathrm{~g}$ material powder was taken, and was extracted using $1.5 \mathrm{~L}$ water by refluxing for $2 \mathrm{~h}$. After filtration, the filtrate was obtained. Then, the residue was extracted using $1500 \mathrm{ml}$ water by refluxing again for $2 \mathrm{~h}$. After filtration, the filtrate was obtained. The filtrates of two extractions were combined, followed by vacuum concentration at $60{ }^{\circ} \mathrm{C}$ to one fifth of the original volume. The $80 \%$ ethanol with quadruple volume to concentrated filtrates was added for precipitation at $4^{\circ} \mathrm{C}$ for $12 \mathrm{~h}$, followed by centrifugal separation. After washing with absolute ethanol and vacuum drying, the crude polysaccharides were obtained. The crude polysaccharides were dissolved in water, and were loaded in the AB- 8 macroporous adsorption resin column, followed by distilling with $20 \%$ ethanol solution. The target elution effluent was collected. After concentration and vacuum drying, the final CPP product was obtained. The content of polysaccharide was $68.12 \%$.

\subsection{Establishment of T2DM model and grouping of rats}

Sixty rats were single-cage raised in the condition avoiding strong light and noise (temperature $22 \pm 2{ }^{\circ} \mathrm{C}$; humidity $40-70 \%$; $12 / 12$-h day-night cycle; free to feed and water). After one week of adaptive feeding, 10 rats were randomly selected as the control group, which were fed with standard diet. The remaining 50 rats were fed with high-fat and high-sugar diet (20\% sucrose, $18 \%$ lard, 3\% yolk and 59\% basic diet) for 4 weeks. The rats were fasted for $12 \mathrm{~h}$, followed by single intraperitoneal injection of streptozotocin solution with dose of $30 \mathrm{mg} / \mathrm{kg}$. After $72 \mathrm{~h}$, the blood sample was taken from tail vein, and the fasting blood glucose $(\mathrm{FBG})$ was detected. The FBG $>16.7 \mathrm{mmol} / \mathrm{L}$ presented the successful establishment of T2DM model. According to FBG value, 40 rats were selected and were randomly divided into model group, CPP-1 group, CPP- 2 group and CPP-3 group, with 10 rats in each group.

\subsection{Treatment of rats}

From the second day after establishment of T2DM model, the rats in CPP-1, CPP-2 and CPP-3 group were intragastrically administrated with CPP, and the dose was 100, 200 and $400 \mathrm{mg} / \mathrm{kg}$, respectively. The rats in control and model group were intragastrically administrated with equal volume of water. The administration was performed once per day, and was lasted for 4 weeks. During the treatment, the body weight, mind state, response, activity, diet and excretion were observed.

\subsection{Observation indexes}

No rat died during the experiment. At the end of treatment, the rats were fasted for $10 \mathrm{~h}$. The blood was collected from abdominal aorta under anesthesia. The FBG level was measured by glucose oxidase method (Thompson, 1966). The fasting insulin (FINS) level was determined by radioimmunoassay. The glycosylated hemoglobin $(\mathrm{GHb})$ and blood lipid indexes including total cholesterol (TC), triglyceride (TG) and low-density lipoprotein cholesterol (LDL-C) were determined using BK-200 automatic biochemical analyzer (Shandong Boke Biological Industry Co.,
Ltd., Jinan, China). The C-reactive protein (CRP) was determined by immunoscattering turbidimetry. The tumor necrosis factor $\alpha$ (TNF- $\alpha$ ) and interleukin 6 (IL-6) were measured by enzyme-linked immunosorbent assay (ELISA). The malondialdehyde (MDA) was determined using thiobarbituric acid method. The superoxide dismutase (SOD) was determined using xanthine oxidase method. The glutathione peroxidase (GSH-Px) was measured by reduced glutathione depletion method. All procedures were in accordance to the instructions of corresponding kits.

\subsection{Statistical analysis}

Data were presented as mean \pm standard deviation. SAS software (version 9.4; SAS Institute Inc., NC, USA) was used for statistical analysis. Multiple comparisons were performed using a one-way analysis of variance followed by the Student-Newman-Keuls test. $\mathrm{P}<0.05$ was considered as statistically significant.

\section{Results}

\subsection{General situation of rats}

During the experiment, the rats in the control group had good spirits and glossy fur, with sensitive response and normal activities. Compared with control group, the rats in the model group had poor mental state, slow response, dull fur, drowsiness, and decreased activities. In addition, they had increased water intake, increased urine volume and loose stool. The rats in CPP-1, CPP-2 and CPP-3 groups also had similar symptoms, but the degree was lighter than the model group.

\subsection{Body weight of rats}

In control group, the body weight of rats sustained a stable growth during the experiment. Compared with control group, the body weight of rats in model, CPP-1, CPP-2 and CPP-3 groups was significantly reduced at $0,1,2,3$ and 4 week time point in treatment, respectively $(\mathrm{P}<0.05)$. The body weight of rats in CPP-2 group at the 3 and 4 week time point and that in CPP-3 groups at the 2, 3 and 4 week time point were significantly higher than that in model group, respectively $(\mathrm{P}<0.05)$ (Figure 1$)$.

\subsection{Blood glucose indexes}

At the end of experiment, the FBG, FINS and GHb levels in model, CPP-1, CPP-2 and CPP-3 groups were significantly higher than those in model group, respectively $(\mathrm{P}<0.05)$. Compared with model group, the FBG and FINS levels in CPP-2 and CPP-3 groups and $\mathrm{GHb}$ level in $\mathrm{CPP}-3$ group were significantly reduced, respectively $(\mathrm{P}<0.05)$ (Table 1$)$.

\subsection{Blood lipid indexes}

After treatment for 4 weeks, the levels of TC, TG and LDL-C in model, CPP-1, CPP-2 and CPP-3 groups were remarkably higher than those in control group, respectively $(\mathrm{P}<0.05)$. Compared with model group, the levels of TC and TG in CPP-3 group and the level of LDL-C in CPP-2 and CPP-3 groups were remarkably decreased, respectively $(\mathrm{P}<0.05)$ (Table 2$)$. 


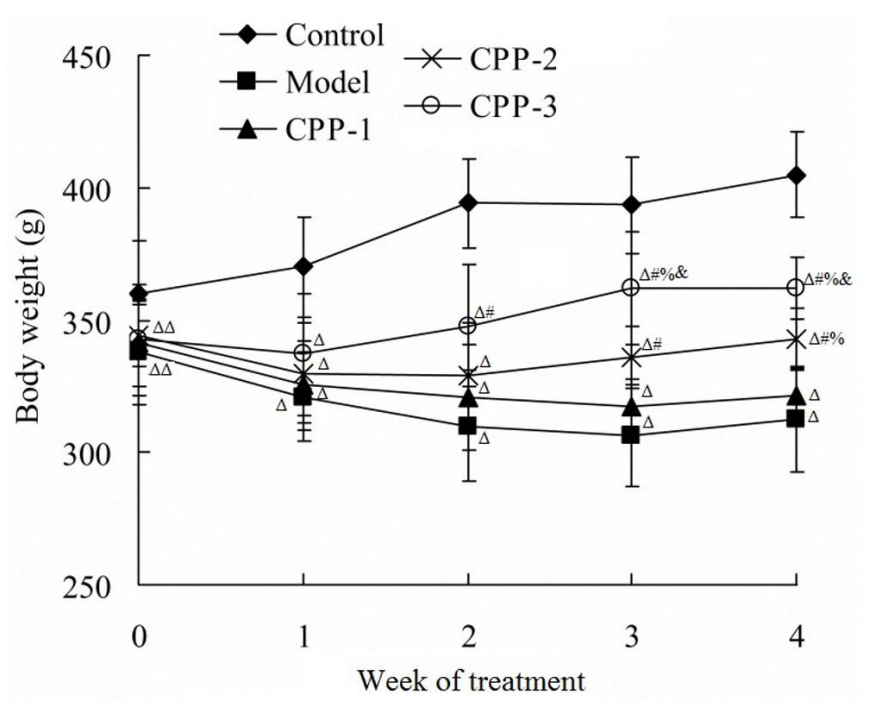

Figure 1. Body weight of rats in different groups. ${ }^{\Delta} \mathrm{P}<0.05 v s$. control group; ${ }^{*} \mathrm{P}<0.05$ vs. model group; ${ }^{\%} \mathrm{P}<0.05$ vs. $\mathrm{CPP}-1$ group; ${ }^{\&} \mathrm{P}<0.05$ vs. CPP-2 group.

Table 1. Blood glucose indexes in different groups.

\begin{tabular}{cccl}
\hline Group & FBG $(\mathrm{mmol} / \mathrm{L})$ & FINS $(\mu \mathrm{U} / \mathrm{L})$ & $\mathrm{GHb}(\%)$ \\
\hline Control & $6.0 \pm 0.8$ & $82.1 \pm 9.2$ & $4.9 \pm 0.8$ \\
Model & $23.1 \pm 4.6^{\Delta}$ & $127.6 \pm 23.1^{\Delta}$ & $9.9 \pm 1.5^{\Delta}$ \\
CPP-1 & $20.2 \pm 5.5^{\Delta}$ & $120.6 \pm 20.2^{\Delta}$ & $9.5 \pm 1.2^{\Delta}$ \\
CPP-2 & $18.3 \pm 3.6^{\Delta *}$ & $108.5 \pm 12.1^{\Delta *}$ & $8.9 \pm 1.0^{\Delta}$ \\
CPP-3 & $15.5 \pm 2.9^{\Delta \sharp \%}$ & $101.4 \pm 14.9^{\Delta \# \%}$ & $6.7 \pm 0.9^{\sharp \% \&}$ \\
\hline
\end{tabular}

${ }^{\Delta} \mathrm{P}<0.05$ vs. control group; ${ }^{"} \mathrm{P}<0.05$ vs. model group; ${ }^{\circ} \mathrm{P}<0.05$ vs. $\mathrm{CPP}-1$ group; ${ }^{\&} \mathrm{P}<0.05$ vs. CPP-2 group. FBG, fasting plasma glucose; FINS, fasting insulin; GHb, glycosylated hemoglobin.

Table 2. Blood lipid indexes in different groups.

\begin{tabular}{cccc}
\hline Group & TC $(\mathrm{mmol} / \mathrm{L})$ & TG $(\mathrm{mmol} / \mathrm{L})$ & LDL-C $(\mathrm{mmol} / \mathrm{L})$ \\
\hline Control & $0.9 \pm 0.2$ & $0.4 \pm 0.1$ & $0.2 \pm 0.0$ \\
Model & $1.7 \pm 0.2^{\Delta}$ & $0.9 \pm 0.1^{\Delta}$ & $0.4 \pm 0.1^{\Delta}$ \\
CPP-1 & $1.6 \pm 0.2^{\Delta}$ & $0.8 \pm 0.1^{\Delta}$ & $0.3 \pm 0.1^{\Delta}$ \\
CPP-2 & $1.4 \pm 0.2^{\Delta}$ & $0.7 \pm 0.2^{\Delta}$ & $0.2 \pm 0.0^{\Delta \sharp}$ \\
CPP-3 & $1.3 \pm 0.2^{\Delta \sharp}$ & $0.5 \pm 0.1^{\Delta \sharp \%}$ & $0.2 \pm 0.0^{\Delta \sharp}$
\end{tabular}

${ }^{4} \mathrm{P}<0.05$ vs. control group; ${ }^{*} \mathrm{P}<0.05$ vs. model group; ${ }^{\circ} \mathrm{P}<0.05$ vs. CPP-1 group. TC, total cholesterol; TG, triglyceride; LDL-C, low-density lipoprotein cholesterol.

\subsection{Levels of inflammatory factors}

Figure 2 showed that, after treatment, the serum CRP, TNF- $\alpha$ and IL-6 levels in model, CPP-1, CPP-2 and CPP-3 groups were remarkably higher than those in normal group, respectively $(\mathrm{P}<0.05)$. Compared with model group, the level of CRP in CPP-1, CPP-2 and CPP-3 groups and the levels of TNF- $\alpha$ and IL-6 in CPP-2 and CPP-3 groups were remarkably reduced, respectively $(\mathrm{P}<0.05)$.

\subsection{Levels of oxidative stress indexes}

After treatment, when comparing with the control group, the serum MDA level in model, CPP-1 and CPP-2 groups was remarkably increased $(\mathrm{P}<0.05)$, and the serum SOD and SOD levels in model, CPP-1, CPP-2 and CPP-3 groups were remarkably decreased, respectively $(\mathrm{P}<0.05)$. Compared with model group, the MDA level in CPP-2 and CPP-3 groups was remarkably reduced, respectively $(\mathrm{P}<0.05)$, and the SOD level in CPP-1, CPP-2 and CPP-3 groups and GSH-Px level in model, CPP-1, CPP-2 and CPP-3 groups were remarkably increased, respectively $(\mathrm{P}<0.05)$ (Table 3$)$.

\section{Discussion}

This study has established the T2DM model in rats, and investigated the effect of CPP on the glucose-lipid metabolism, inflammatory response and oxidative stress in T2DM rats. Results showed that, compared with model group, in CPP treatment groups the T2DM symptoms of rats were lightened, the body weight was improved, and the FBG, FINS and GHb levels were reduced. This indicates that, $\mathrm{CPP}$ can obviously ease the diabetic symptoms and reduce the blood glucose level.

Table 3. Levels of oxidative stress indexes in different groups.

\begin{tabular}{cccc}
\hline Group & MDA $(\mu \mathrm{mol} / \mathrm{L})$ & SOD $(\mathrm{U} / \mathrm{ml})$ & GSH-Px $(\mathrm{U} / \mathrm{ml})$ \\
\hline Control & $0.3 \pm 0.1$ & $27.3 \pm 4.0$ & $1022.7 \pm 136.7$ \\
Model & $0.5 \pm 0.0^{\Delta}$ & $12.6 \pm 1.8^{\Delta}$ & $707.6 \pm 98.6^{\Delta}$ \\
CPP-1 & $0.5 \pm 0.1^{\Delta}$ & $14.6 \pm 1.9^{\Delta *}$ & $714.7 \pm 93.3^{\Delta}$ \\
CPP-2 & $0.4 \pm 0.1^{\Delta *}$ & $17.3 \pm 2.0^{\Delta * \%}$ & $806.7 \pm 101.4^{\Delta * \%}$ \\
CPP-3 & $0.3 \pm 0.1^{\# \% \&}$ & $18.5 \pm 1.2^{\Delta * \%}$ & $887.5 \pm 93.6^{\Delta * \%}$ \\
\hline
\end{tabular}

${ }^{\Delta} \mathrm{P}<0.05$ vs. control group; ${ }^{\mathrm{P}}<0.05$ vs. model group; ${ }^{\circ} \mathrm{P}<0.05$ vs. CPP-1 group. MDA, malondialdehyde; SOD, superoxide dismutase. GSH-Px, glutathione peroxidase.
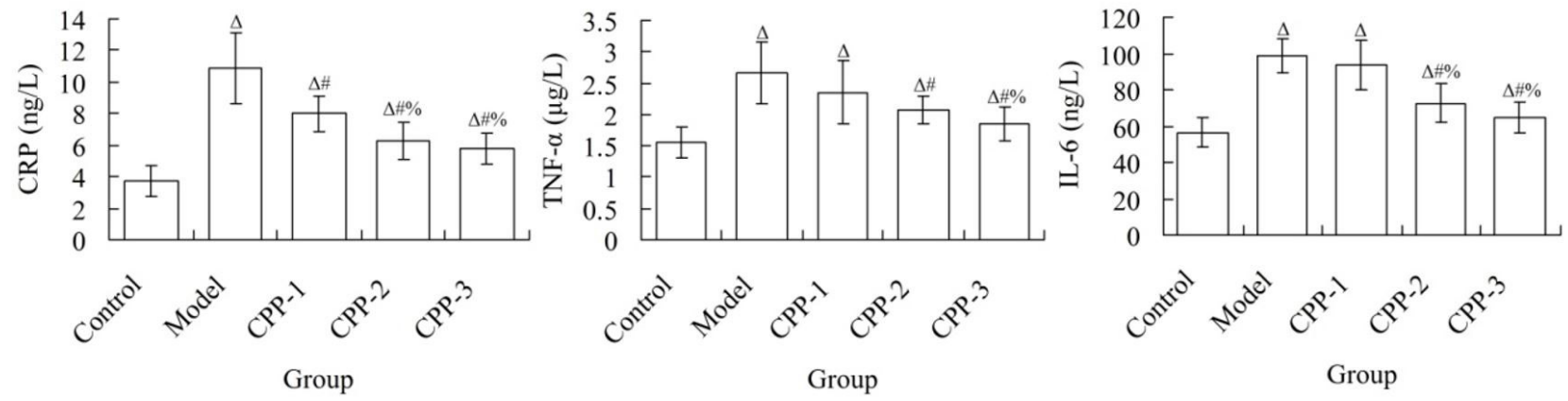

Figure 2. Levels of inflammatory factors in different groups. ${ }^{\triangle} \mathrm{P}<0.05$ vs. control group; ${ }^{\sharp} \mathrm{P}<0.05$ vs. model group; ${ }^{\circ} \mathrm{P}<0.05$ vs. CPP-1 group. CRP, C-reactive protein; TNF- $\alpha$, tumor necrosis factor $\alpha$; IL-6, interleukin 6. 
It is found that, high blood lipid levels are closely related to the occurrence and development of T2DM. TG is the storage form of free fatty acids (FFA) in tissues before hydrolysis, while FFA is the hydrolysis product of TG (Ho et al., 2002). When the FFA level increases, this exceeds the fat storage capacity and oxidative capacity of tissues to FFA. The FFA deposits in non-adipose tissues in the form of TG, which results in tissue damage (Bays et al., 2004; Ma et al., 2014). For example, excessive TG deposit in the islets can accelerate the apoptosis of islet $\beta$ cells and decrease their function (Cnop et al., 2001). Results of this study showed that, compared with model group, the levels of TC and TG in CPP-3 group and the level of LDL-C in CPP-2 and CPP-3 groups were remarkably decreased. This suggests that, CPP can reduce the blood lipid levels, thus preventing the development of T2DM in rats.

In recent years, it has been argued that diabetes mellitus and its vascular lesions are the inflammatory diseases. This inflammatory response is considered to be a subclinical inflammation, which is below the level of infectious and autoimmune inflammation. This inflammation further leads to insulin resistance, islet $\beta$ cell injury and vascular endothelial cell dysfunction, thus causing diabetes mellitus and its chronic vascular complications (Peng et al., 2010; Morgan et al., 2014). CRP is the main protein involved in inflammation or acute phase reaction, and is also the most sensitive indicator. CRP is the strongest predictor of the onset of T2DM. Studies have found that the CRP level increases significantly in newly discovered or diagnosed diabetes mellitus patients (Qiu et al., 2004). TNF- $\alpha$ is a polypeptide mediator produced by mononuclear macrophages. It is found that TNF- $\alpha$ plays an important role in the pathogenesis of insulin resistance, and is closely related to plasma insulin level, glucose metabolism and lipid metabolism (Mueller et al., 1993). IL-6 is another inflammatory factor closely related to the pathogenesis of diabetes mellitus. It is a promoter of insulin function under normal conditions and a damage factor under pathological conditions. The increased IL- 6 may damage the function of $\beta$ cells and inhibit the insulin secretion, thus promoting the development of T2DM (Watt et al., 2005). Results of this study showed that, after treatment, compared with model group, the level of CRP in CPP-1, CPP-2 and CPP-3 groups and the levels of TNF- $\alpha$ and IL- 6 in CPP-2 and CPP-3 groups were remarkably reduced. This indicates that, $\mathrm{CPP}$ can decrease the CRP, TNF- $\alpha$ and IL- 6 levels and reduce the inflammatory response, which decreases the insulin resistance and dysfunction of $\beta$ cells, thus alleviating the T2DM in rats.

Oxidative stress refers to the imbalance between the production of reactive oxygen species (ROS) and the antioxidant defense system in body, which leads to tissue damage. Under normal physiological conditions, ROS produced by oxidative stress can be quickly eliminated by antioxidant system. However, the hyperglycemia causes the production of excessive ROS in mitochondria of vascular endothelial cells, which aggravates the oxidative stress reaction in cells (Wolff et al., 1991; Singh et al., 2009). SOD, as one of the main antioxidants, is ubiquitous in metabolic cells. Study has confirmed that there is metabolic disorder of SOD in patients with T2DM, which lead to decreased anti-peroxidation ability and increased free radical production (Mayhan, 1997). GSH-Px is an important catalytic oxidase widely existing in the body. It can also scavenge free radicals, block the chain reaction of lipid peroxidation and protect the structure and function of cell membrane. The level of GSH-Px is decreased in T2DM diabetic patients (Kornhauser et al., 2008) and T2DM rat model (Zhu et al., 2016). Oxygen free radicals destroy the structure and function of cells by lipid peroxidation with unsaturated fatty acids in cell membranes, and MDA is formed at the same time (Arslan et al., 2014). Results of this study showed that, compared with model group, the MDA level in CPP-2 and CPP-3 groups was remarkably reduced, and the SOD level in CPP-1, CPP-2 and CPP-3 groups and GSH-Px level in model, CPP-1, CPP-2 and CPP-3 groups were remarkably increased. This suggests that, CPP has the ability of scavenging radical and reducing lipid peroxidation, thus playing a protection role for $\mathrm{T} 2 \mathrm{DM}$ in rats.

\section{Conclusion}

CPP can alleviate the T2DM in rats, which may be related to its resisting inflammatory response and oxidative stress in body. This study has provided an experimental basis for studying the clinical therapeutic effects of CPP on T2DM. However, the other effects of CPP on T2DM rats need to be further investigated.

\section{References}

Arslan, M., Ipekci, S. H., Kebapcilar, L., Dogan Dede, N., Kurban, S., Erbay, E., \& Gonen, M. S. (2014). Effect of aerobic exercise training on MDA and TNF- $\alpha$ levels in patients with type 2 diabetes mellitus. International Scholarly Research Notices, 2014, 820387. http://dx.doi. org/10.1155/2014/820387. PMid:27437465.

Bays, H., Mandarino, L., \& DeFronzo, R. A. (2004). Role of the adipocyte, free fatty acids, and ectopic fat in pathogenesis of type 2 diabetes mellitus: peroxisomal proliferator-activated receptor agonists provide a rational therapeutic approach. The Journal of Clinical Endocrinology and Metabolism, 89(2), 463-478. http://dx.doi.org/10.1210/jc.2003030723. PMid:14764748.

Cavaghan, M. K., Ehrmann, D. A., \& Polonsky, K. S. (2000). Interactions between insulin resistance and insulin secretion in the development of glucose intolerance. The Journal of Clinical Investigation, 106(3), 329-333. http://dx.doi.org/10.1172/JCI10761. PMid:10930434.

Cnop, M., Hannaert, J. C., Hoorens, A., Eizirik, D. L., \& Pipeleers, D. G. (2001). Inverse relationship between cytotoxicity of free fatty acids in pancreatic islet cells and cellular triglyceride accumulation. Diabetes, 50(8), 1771-1777. http://dx.doi.org/10.2337/diabetes.50.8.1771. PMid:11473037.

Diakakis, G. F., Parthenakis, F. I., Mavrakis, H. E., Patrianakos, A. P., Zacharis, E. A., Nyktari, E. G., Ganotakis, E. S., \& Vardas, P. E. (2005). Association of impaired glucose tolerance with increased heart rate and subclinical inflammation. Hellenic Journal of Cardiology, 46(6), 394-401. PMid:16422126.

Ho, S. Y., Delgado, L., \& Storch, J. (2002). Monoacylglycerol metabolism in human intestinal Caco-2 cells: evidence for metabolic compartmentation and hydrolysis. The Journal of Biological Chemistry, 277(3), 18161823. http://dx.doi.org/10.1074/jbc.M108027200. PMid:11682480.

Huang, J. Y., Lou, L. Y., \& Xu, P. (1986). Studies on the pharmacology of the leaves of Cyclocarya paliurus. Zhongguo Zhongyao Zazhi, 11, 61-63. [in Chinese]

Kornhauser, C., Garcia-Ramirez, J. R., Wrobel, K., Pérez-Luque, E. L., Garay-Sevilla, M. E., \& Wrobel, K. (2008). Serum selenium 
and glutathione peroxidase concentrations in type 2 diabetes mellitus patients. Primary Care Diabetes, 2(2), 81-85. http://dx.doi. org/10.1016/j.pcd.2008.02.003. PMid:18684427.

Kurihara, H., Fukami, H., Kusumoto, A., Toyoda, Y., Shibata, H., Matsui, Y., Asami, S., \& Tanaka, T. (2003). Hypoglycemic action of Cyclocarya paliurus (Batal.) Iljinskaja in normal and diabetic mice. Bioscience, Biotechnology, and Biochemistry, 67(4), 877-880. http:// dx.doi.org/10.1271/bbb.67.877. PMid:12784631.

Ma, Y., Wang, Y., Huang, Q., Ren, Q., Chen, S., Zhang, A., Zhao, L., Zhen, Q., \& Peng, Y. (2014). Impaired $\beta$ cell function in Chinese newly diagnosed type 2 diabetes mellitus with hyperlipidemia. Journal of Diabetes Research, 2014, 493039. http://dx.doi.org/10.1155/2014/493039. PMid:24829924.

Mayhan, W. G. (1997). Superoxide dismutase partially restores impaired dilatation of the basilar artery during diabetes mellitus. Brain Research, 760(1-2), 204-209. http://dx.doi.org/10.1016/S0006-8993(97)002825. PMid:9237536.

Morgan, N. G., Leete, P., Foulis, A. K., \& Richardson, S. J. (2014). Islet inflammation in human type 1 diabetes mellitus. IUBMB Life, 66(11), 723-734. http://dx.doi.org/10.1002/iub.1330. PMid:25504835.

Mueller, C., Imboden, M. A., Hess, M. W., Laissue, J. A., \& Carnaud, C. C. (1993). TNF- $\alpha$ and insulin-dependent diabetes mellitus. Research in Immunology, 144(5), 331-335. http://dx.doi.org/10.1016/S09232494(93)80076-B. PMid:8278654.

Nan, L.Y., Jing, Z., Ru, W., Zhan-wei, Y., \& Wen-jun, W. (2015). Effects of Cyclocarya paliurus (Batal.) Iljinskaja polysaccharides on lipid metabolism and expressions of PPARa, FAS, and GLUT4 mRNA in hyperlipidemic rats. Xiandai Shipin Keji, 31, 29-35. [in Chinese]

Olokoba, A. B., Obateru, O. A., \& Olokoba, L. B. (2012). Type 2 diabetes mellitus: a review of current trends. Oman Medical Journal, 27(4), 269-273. http://dx.doi.org/10.5001/omj.2012.68. PMid:23071876.

Peng, H., Olsen, G., Tamura, Y., Noguchi, H., Matsumoto, S., Levy, M. F., \& Naziruddin, B. (2010). Inhibition of inflammatory cytokine-induced response in human islet cells by withaferin A. Transplantation Proceedings, 42(6), 2058-2061. http://dx.doi. org/10.1016/j.transproceed.2010.05.131. PMid:20692407.

Qiu, C., Sorensen, T. K., Luthy, D. A., \& Williams, M. A. (2004). A prospective study of maternal serum C-reactive protein (CRP) concentrations and risk of gestational diabetes mellitus. Paediatric and Perinatal Epidemiology, 18(5), 377-384. http://dx.doi.org/10.1111/ j.1365-3016.2004.00578.x. PMid:15367325.

Rehman, K., \& Akash, M. S. H. (2017). Mechanism of generation of oxidative stress and pathophysiology of type 2 diabetes mellitus: how are they interlinked? Journal of Cellular Biochemistry, 118(11), 3577-3585. http://dx.doi.org/10.1002/jcb.26097. PMid:28460155.

Ruotsalainen, E., Salmenniemi, U., Vauhkonen, I., Pihlajamäki, J., Punnonen, K., Kainulainen, S., \& Laakso, M. (2006). Changes in inflammatory cytokines are related to impaired glucose tolerance in offspring of type 2 diabetic subjects. Diabetes Care, 29(12), 2714-2720. http://dx.doi.org/10.2337/dc06-0147. PMid:17130210.
Singh, P. P., Mahadi, F., Roy, A., \& Sharma, P. (2009). Reactive oxygen species, reactive nitrogen species and antioxidants in etiopathogenesis of diabetes mellitus type-2. Indian Journal of Clinical Biochemistry, 24(4), 324-342. http://dx.doi.org/10.1007/s12291-009-0062-6. PMid:23105858.

Tangvarasittichai, S. (2015). Oxidative stress, insulin resistance, dyslipidemia and type 2 diabetes mellitus. World Journal of Diabetes, 6(3), 456-480. http://dx.doi.org/10.4239/wjd.v6.i3.456. PMid:25897356.

Tatsch, E., Carvalho, J. A. M. D., Hausen, B. S., Bollick, Y. S., Torbitz, V. D., Duarte, T., Scolari, R., Duarte, M. M. M. F., Londero, S. W. K., Vaucher, R. A., Premaor, M. O., Comim, F. V., \& Moresco, R. N. (2015). Oxidative DNA damage is associated with inflammatory response, insulin resistance and microvascular complications in type 2 diabetes. Mutation Research, 782, 17-22. http://dx.doi.org/10.1016/j. mrfmmm.2015.10.003. PMid:26520687.

Thompson, R. H. (1966). Colorimetric glucose oxidase method for blood glucose. Clinica Chimica Acta: International Journal of Clinical Chemistry, 13(1), 133-135. http://dx.doi.org/10.1016/00098981(66)90281-6. PMid:5917676.

van Doorn, M., Kemme, M., Ouwens, M., van Hoogdalem, E. J., Jones, R., Romijn, H., de Kam, M., Schoemaker, R., Burggraaf, K., \& Cohen, A. (2006). Evaluation of proinflammatory cytokines and inflammation markers as biomarkers for the action of thiazolidinediones in Type 2 diabetes mellitus patients and healthy volunteers. British Journal of Clinical Pharmacology, 62(4), 391-402. http://dx.doi. org/10.1111/j.1365-2125.2005.02532.x. PMid:16995860.

Watt, M. J., Carey, A. L., Wolsk-Petersen, E., Kraemer, F. B., Pedersen, B. K., \& Febbraio, M. A. (2005). Hormone-sensitive lipase is reduced in the adipose tissue of patients with type 2 diabetes mellitus: influence of IL-6 infusion. Diabetologia, 48(1), 105-112. http:// dx.doi.org/10.1007/s00125-004-1598-x. PMid:15609025.

Wolff, S. P., Jiang, Z. Y., \& Hunt, J. V. (1991). Protein glycation and oxidative stress in diabetes mellitus and ageing. Free Radical Biology \& Medicine, 10(5), 339-352. http://dx.doi.org/10.1016/08915849(91)90040-A. PMid:1855674.

Xie, J. H., Shen, M. Y., Nie, S. P., Zhao, Q., Li, C., \& Xie, M. Y. (2014). Separation of water-soluble polysaccharides from Cyclocarya paliurus by ultrafiltration process. Carbohydrate Polymers, 101, 479-483. http://dx.doi.org/10.1016/j.carbpol.2013.09.075. PMid:24299801.

Xie, J. H., Xie, M. Y., Shen, M. Y., Nie, S. P., Li, C., \& Wang, Y. X. (2010). Optimisation of microwave-assisted extraction of polysaccharides from Cyclocarya paliurus (Batal.) Iljinskaja using response surface methodology. Journal of the Science of Food and Agriculture, 90(8), 1353-1360. http://dx.doi.org/10.1002/jsfa.3935. PMid:20474055.

Zhu, H., Wang, Y., Liu, Z., Wang, J., Wan, D., Feng, S., Yang, X., \& Wang, T. (2016). Antidiabetic and antioxidant effects of catalpol extracted fromRehmannia glutinosa (Di Huang) on rat diabetes induced by streptozotocin and high-fat, high-sugar feed. Chinese Medicine, 11(1), 25. http://dx.doi.org/10.1186/s13020-016-0096-7. PMid:27175212. 